Cahiers $d u$ MONDE RUSSE

\section{Cahiers du monde russe}

Russie - Empire russe - Union soviétique et États indépendants

$49 / 4 \mid 2008$

Destins individuels et terreur. Jeunesse dans la société post-stalinienne

\title{
Birgit Beumers, ed., The Cinema of Russia and the Former Soviet Union
}

\section{Martine Godet}

\section{Q OpenEdition}

\section{Journals}

Édition électronique

URL : https://journals.openedition.org/monderusse/6964

DOI : 10.4000/monderusse. 6964

ISSN : $1777-5388$

Éditeur

Éditions de l'EHESS

\section{Édition imprimée}

Date de publication : 28 décembre 2008

Pagination : 776-781

ISBN : 978-2-7132-2197-2

ISSN : $1252-6576$

Référence électronique

Martine Godet, «Birgit Beumers, ed., The Cinema of Russia and the Former Soviet Union », Cahiers du monde russe [En ligne], 49/4 | 2008, mis en ligne le 24 décembre 2009, consulté le 03 septembre 2022. URL : http://journals.openedition.org/monderusse/6964 ; DOI : https://doi.org/10.4000/ monderusse.6964

Ce document a été généré automatiquement le 3 septembre 2022.

Tous droits réservés 


\title{
Birgit Beumers, ed., The Cinema of Russia and the Former Soviet Union
}

\author{
Martine Godet
}

\section{RÉFÉRENCE}

Birgit Beumers, ed., The Cinema of Russia and the Former Soviet Union. Préface de Sergei Bodrov Sr. Londres : Wallflower, 2007, 283 p. (Series « 24 Frames »)

1 Attachée au Département de russe de l'université de Bristol, directrice du Centre for Russian and East European Cultural Studies, Birgit Beumers est spécialiste de la culture russe contemporaine au sens large. Après une thèse consacrée à Jurij Ljubimov au théâtre de la Taganka ${ }^{1}$, Beumers s'est ensuite tournée vers le cinéma ${ }^{2}$ et fédère actuellement autour d'elle un courant de recherche anglo-saxon qui se place dans l'optique des «cultural studies » et de la sémiologie, et participe très largement au renouveau historiographique du cinéma russe actuel. Elle est notamment l'éditrice de Kinokultura, revue en ligne très active.

2 Beumers a dirigé ce livre collectif qui est composé de 24 analyses de film, selon le principe de la collection dans laquelle il s'insère. Le résultat est particulièrement rafraîchissant par l'alternance de films très célèbres et d'œuvres plus rares, d'auteurs chevronnés et d'autres moins connus. Dans cet ensemble, qui va du cinéma muet d'avant la révolution jusqu'à la période postsoviétique incluse et dont il serait impossible faute de place, voire fastidieux, de rendre compte exhaustivement, nous opérerons une sélection à partir du Dégel, en favorisant la période la plus contemporaine à laquelle une large place est réservée dans l'ouvrage.

3 Nuit de carnaval (Karnaval'naja noč'), réalisé en 1956, première comédie d'El'dar Rjazanov le cinéaste le plus populaire de Russie - est l'un des films précurseurs du Dégel, l'un des premiers à s'écarter vraiment du stalinisme. En tête de la programmation, il a été vu par 48640000 spectateurs l'année de sa sortie ! De jeunes employés d'une grande usine sont chargés par le directeur de la Maison de la culture de préparer la soirée du Nouvel An. La 
touche "carnaval» ajoutée au titre du film implique une ambiance de fête, un échappatoire, un dérèglement par rapport à la norme: il s'agira donc d'un événement exceptionnel, et non d'un simple réveillon. Les jeunes se mettent au travail avec talent et enthousiasme, débordant de rires et de vie, sous la direction de Lena Krylova (premier rôle de l'actrice Ljudmila Gurčenko). Le traitement du film n'a rien à voir avec le réalisme socialiste - on sent les prémices du Dégel avec l'ébauche d'un retour à l'individu, à l'émotion vraie, la mise en scène d'une histoire d'amour privée entre Lena et son amoureux Griša. Ce qui est nouveau également, c'est le traitement des situations sur le mode de la légèreté et de l'humour, la relativité des valeurs. La chanson joue un grand rôle dans le film, faisant de celui-ci l'héritier des comédies musicales staliniennes de Grigorij Aleksandrov et d'Ivan Pyr'ev, mais dans un genre totalement renouvelé.

4 Néanmoins, la jeune génération se heurte, dans son désir d'innovation, au directeur de la Maison de la culture, le bien-nommé Ogurcov, qui bloque toute initiative novatrice (et qui finira la soirée enfermé dans un placard). Car l'objectif qui sous-tend le film est une critique de la bureaucratie soviétique, encouragée d'ailleurs officiellement à l'époque khrouchtchevienne. S'y ajoute en filigrane celle de l'intervention abusive du pouvoir politique dans le domaine de l'art. Mais le conflit de générations est traité avec drôlerie, arme majeure de Rjazanov tout au long de sa carrière. La comédie, même satirique, est un genre très populaire qui échappe davantage que le cinéma d'auteur à la surveillance, car moins suspect de subversivité, sans compter qu'il remplit les caisses de l'État, argument non négligeable. À l'époque suivante, Rjazanov se dérobera ainsi à la censure grâce à l'arme du rire, pour des comédies très critiques comme Garage (Garaž, 1980). Il faut signaler enfin une analyse très élaborée de la forme filmique par David MacFayden (p. 89-97).

5 En ce qui concerne la période brejnévienne, plutôt que de traiter des deux films censurés qui sont analysés dans le livre³, Brèves rencontres (Korotkie vstreči) de Kira Muratova (1967), par Susan Larsen (p. 119-127), et Mon ami Ivan Lapšin (Moj drug Ivan Lapšin) d'Aleksej German (1984), par Anthony Anemone (p. 203-211) ${ }^{4}$, nous retiendrons ici une œuvre peu connue mais particulièrement intéressante de Nikita Mihalkov. Il s'agit de son premier long-métrage, Ami chez les ennemis, ennemi chez les amis (Svoj sredi čužih, čužoj sredi svoih), réalisé en $1974^{5}$. L'action se déroule au début des années 1920, juste après la fin de la guerre civile, dans le sud de la Russie. L'intrigue est très complexe, croisant Rouges, Blancs et Verts (anarchistes). Les cinq protagonistes du film, tchékistes, qui ont tous appartenu à la Cavalerie rouge, sont chargés de protéger un convoi qui transporte à Moscou l'or pris aux Blancs. Or le convoi est attaqué et l'or confisqué. L'un de ces cinq hommes, resté en vie, est accusé de trahison. Une véritable ambiance de suspicion paranoïaque règne au Quartier général des Rouges. L'homme accusé part seul à la recherche du trésor perdu. Il finira par retrouver l'or à force de courage et de ruse, et à prouver ainsi son innocence.

6 Sur le plan du récit et de la forme, le film est conçu comme un western - version western spaghetti, proche du film de Sergio Leone, Le bon, la brute et le truand (1966) - mais un western « de l'Est », comme l'analyse Elena Prokhorova (p. 171-180). Il appartiendrait à la longue tradition des récits et des films d'aventure soviétiques sur la guerre civile qui auraient un rapport, dans l'imaginaire des Russes, avec la conquête de l'Ouest américain. L'auteur ébauche une comparaison avec Čapaev, 1934, des Frères Vasil'ev, ou Le soleil blanc du désert (Vladimir Motyl', 1969). On pourrait évoquer Pas de gué dans le feu (V ogne broda 
net, Gleb Panfilov, 1967), où l'on retrouve la même complexité d'intrigue, ou encore Le Communiste (Kommunist, Julij Raizman, 1957).

7 Prokhorova recadre le film dans l'ensemble de l'œuvre de Mihalkov, évoquant la période trouble des débuts de l'époque brejnévienne (le film est entamé dès 1971 à Mosfilm). Malgré une bonne réception auprès du public (23,7 millions de spectateurs en 1974), les critiques sur le film sont mitigées. De fait, bien qu'il suive le dogme officiel sur l'histoire de la guerre civile - au sens où les Blancs et les Verts remplissent la fonction narrative d'anti-héros indispensable pour restaurer la figure d'un héros fort dans les temps non héroïques de l'ère de stagnation -, le conflit entre confiance et trahison est traité comme un dilemme privé et non comme un drame historique. La complexité même de l'intrigue est éloignée des canons simples du héros positif, sans compter la confusion des valeurs, objectifs et idéaux, spécifique de l'ère de stagnation. La multiplication des flashbacks pour évoquer le passé confère par ailleurs à celui-ci une connotation de paradis perdu et casse l'utopie soviétique en suggérant la perte d'un monde idéal et de la fraternité révolutionnaire. De fait, ce film constitue un exemple type d'œuvre de la période brejnévienne que son message ambigu aurait pu faire basculer vers la censure si son jeune réalisateur n'avait pas bénéficié d'un certain nombre de soutiens.

8 Pour la période de la perestroïka, un seul film est analysé dans l'ouvrage, mais il s'agit d'une œuvre essentielle, L'Aiguille (Igla) de Rašid Nugmanov (1988), étudié par Birgit Beumers (p. 213-221). L'un des représentants de la « nouvelle vague kazakh », Nugmanov a été formé au VGIK à partir de 1984 par Sergej Solov'ev en même temps qu'Omirbaev, Aprymov, Amirkulov, Karpykov et Karakulov. Au début des années 1980, le studio Kazahfil'm avait en effet servi de plateforme pour de jeunes cinéastes expérimentaux venant de Moscou, tels Sergej Bodrov et Solov'ev. C'est dans ce cadre que ce dernier avait sélectionné les jeunes espoirs locaux pour les envoyer en formation à Moscou. En 1988, Solov'ev réalise Assa, et son élève, L'Aiguille. Nugmanov reprend de nombreux procédés de son maître, notamment l'accent mis sur la musique rock - réduite à une existence underground à l'époque brejnévienne et symbole du désir de changement de la jeunesse - et le choix de héros non professionnels. Le débutant réalise un film parfaitement équilibré, dans lequel, contrairement à Assa, la partie rock s'intègre de manière homogène dans l'action.

Viktor Coj, leader fétiche du groupe rock Kino, interprète le héros du film, Moro. Il est l'auteur des chansons du film et leur exécutant. Coj incarne alors pour les jeunes Soviétiques, outre un hymne à leur génération, le double rejet du monde corrompu des adultes et du système soviétique ${ }^{6}$. Moro, sorte d'ange purificateur à la beauté saisissante, retourne à Almaty, sa ville natale, pour récupérer l'argent que lui doit un jeune truand minable. Il retrouve à cette occasion son ancienne amie, Dina, et réalise rapidement qu'elle est toxicodépendante et sous la coupe d'un médecin plus âgé qui a monté un réseau de drogue. Pour l'arracher à l'héroïne, Moro l'emmène sur les bords de la mer d'Aral, où ils étaient déjà venus ensemble autrefois. Ils passent quelques jours dans une maison de pêcheurs en ruines, dans un retour aux sources où la désintoxication peut s'opérer. Mais, entretemps, la mer a reculé. Des plans magnifiques montrent leurs silhouettes avançant vers l'horizon, l'écran coupé en deux horizontalement entre le sable très blanc et le ciel bleu intense. Enfin Moro et Dina arrivent à ce bateau échoué qui a servi d'emblème à la désertification de la mer d'Aral. Avant tous les documentaires qui reprendront cette image, Nugmanov cible en une scène, sans un mot, l'immense gâchis environnemental perpétré par les Soviétiques. Il s'agit d'un film d'une grande maturité 
puisque Nugmanov croise l'espoir de changement de la jeune génération, thématique phare de la perestroïka, avec le problème social du développement de la drogue et la stigmatisation du lourd héritage de l'URSS. La forme est d'une impeccable sobriété. C'est l'amorce du nouveau courant cinématographique d'Asie centrale qui va, durant dix à quinze ans, prendre le relais d'un cinéma russe en perdition.

Le Frère (Brat), d'Aleksej Balabanov, est un thriller d'un genre totalement nouveau dans la Russie postsoviétique de 1997, plongée dans l'ère El'cin, où il émerge alors que le cinéma russe est en plein marasme. La production cinématographique s'est effondrée depuis 1992 et a atteint son plus bas niveau (1996); la distribution est inexistante; il n'y a pas de salles pour projeter les films russes. Malgré ce contexte, Brat devient immédiatement une œuvre-culte pour la jeune génération - et, de fait, l'un des seuls films russes que l'on retienne de la fin des années 1990.

11 À peine de retour dans sa ville natale après deux années de service militaire, Danila Bagrov part à Saint-Pétersbourg, où se déroule toute l'action du film, pour rejoindre son frère aîné, tueur à gages. Il trouve un monde corrompu où règne la loi du plus fort, où mafia et racket ont envahi la scène, où la police est largement déconsidérée - et il choisit sa voie, celle de la violence, apprenant vite à transgresser la légalité. Le rôle principal est joué par le jeune acteur Sergej Bodrov Jr. qui s'était fait connaître un an plus tôt dans un film réalisé par son propre père, Prisonnier du Caucase (1996). Bagrov incarne un héros atypique, sorte de justicier dépourvu de principes moraux mais au cœur noble, qui tue pour faire disparaitre la racaille tout en prenant la défense du faible et de l'opprimé. La perte des valeurs, déjà stigmatisée par le cinéma brejnévien, est abyssale dans la Russie de la fin des années 1990 où il n'y a plus de structure familiale : la mère de Danila a toujours préféré son fils aîné, qui se révèle un lâche - il n'y a donc plus de « frère » et la fraternité est à trouver ailleurs; le père est mort en prison quand le cadet était adolescent. La seule personne que Bagrov respecte est l'« Allemand », un vieux mendiant qui a su garder des valeurs éthiques dans la dérive générale et refuse l'argent salement gagné. Le héros, seul dans une société hostile, fuit dans le monde virtuel de la musique rock, qui constitue à nouveau un élément central de la structure du film et lui donne son sens, comme l'a décrypté Birgit Beumers qui signe également l'analyse (p. 233-241).

Beumers insiste sur l'émergence, avec le personnage de Danila Bagrov, d'un nouveau type de " héros russe " positif qui tranche avec la grisaille (černuha) des héros victimes de la perestroïka, ce qui expliquerait le succès immédiat du film. L'auteur se demande dubitativement s'il faut comparer ce retour à la célébration de valeurs nationales avec les principes du réalisme socialiste qui prônaient un vernissage (lakirovka) de la réalité. Beumers avait déjà creusé le thème de " l'idée russe » dans le cinéma postsoviétique ${ }^{7}$ et cible ici les tendances nationalistes du héros en confrontant Brat avec la suite du film, Brat 2 (2000), dont l'action se déroule aux États-Unis. D'un film à l'autre, Danila n'a pas de mots assez durs pour les « mafieux tchétchènes » et les Caucasiens en général, tient des propos racistes contre les "nègres" et pratique un antisémitisme à peine voilé. Néanmoins, à la différence de certains critiques russes qui avaient fustigé cet aspect xénophobe, Beumers fait remonter l'analyse et l'élargit en concluant plutôt à une sorte d'hypernationalisme du réalisateur Balabanov qui « réaffirme Moscou comme centre de l'univers ».

13 L'une des analyses les plus fouillées de l'ouvrage concerne une œuvre extrêmement complexe de la période postsoviétique, La maison des fous (Dom durakov) d'Andrej Končalovskij (2002), étudiée par Marcia Landy (p. 253-262). L'action se déroule dans un 
asile psychiatrique situé à la frontière russo-tchétchène. La vie des patients est gravement perturbée par le conflit. Le film est une allégorie satirique de la guerre de Tchétchénie, une tentative de "démantèlement polémique » des formes de violence qui sous-tendent le conflit. Končalovskij cherche à déstabiliser les notions d'identité nationale, de guerre, d'hérö̈sme et de pouvoir d'État, dans une perspective internationaliste, ou plutôt "cosmopolite ". La maison des fous développe ainsi une attitude critique envers la politique qui relèverait davantage d'une approche cinématographique contemporaine en s'écartant de la conception d'un cinéma national. À contre-courant de la tendance actuelle en Russie.

Plus l'action avance et les faits de guerre se multiplient, plus le film estompe la frontière entre «fous» et sains d'esprit, plus il devient impossible d'établir une frontière enre normal et pathologique. L'asile psychiatrique est un lieu qui prend toutes les propriétés du carnavalesque, offrant une vision du monde grotesque et désordonnée où tout est inversé, sens dessus dessous, dans laquelle le non-sens révèle la tension entre chaos et stabilité. Le monde de l'hôpital devient le microcosme du monde russe. Le comportement outrancier, extravagant, des malades semble plus sain mentalement que le monde dominant du pouvoir et de la politique - stratégie du réalisateur pour déstabiliser la notion qu'a le public d'une réalité fallacieuse qui produit privations, mort et destruction. Le cinéma pourrait-il ainsi contribuer à trouver un langage pour rendre compte de la complexité d'une situation qui résiste aux modes de représentation conventionnels? Néanmoins, Končalovskij - délibérément - ne tranche pas : le film ne résout pas le conflit qu'il contient et n'offre pas de vision rassurante de l'histoire nationale russe.

La méthode d'analyse filmique très élaborée qui est pratiquée dans l'ouvrage engendre indéniablement un résultat remarquable en ce qui concerne la connaissance de l'œuvre elle-même. Notons néanmoins que poussée très loin, cette approche peut parfois aboutir à des interprétations hasardeuses. Les données historiques également sont floues en certaines occurrences. Enfin l'abus des mêmes références bibliographiques que l'on retrouve fréquemment chez les auteurs anglo-saxons - Benjamin, Deleuze, Foucault, parmi d'autres - est un reproche récurrent. Ceci ne doit pas occulter l'essentiel : loin de constituer une somme disparate d'analyses de film éclatées, l'ouvrage brosse un panorama d'ensemble largement novateur du cinéma russe.

\section{NOTES}

1. Yuri Lyubimov at the Taganka Theatre 1964-1994, Amsterdam : Harwood Academic Publishers/ OPA, 1997.

2. B. Beumers a notamment dirigé Russia on Reels: The Russian Idea in Post-Soviet Cinema, Londres : I.B. Tauris, 2000, et publie en 2009 A History of Russian Cinema, Oxford-New York : Berg.

3. Auxquels s'ajoute Le Conte des contes (Skazka skazok), film d'animation de Jurij Norštejn, 1979, analysé ici par David MacFadyen, p. 183-191.

4. Sur cette période, voir aussi: Martine Godet, La pellicule et les ciseaux. La censure dans le cinéma soviétique du Dégel à la perestroika, P. : Éditions du CNRS (ouvrage sous presse). 
5. La toute première œuvre du cinéaste, très prometteuse, étant son film de diplôme, Une journée tranquille à la fin de la guerre (Spokojnyj den' v konce vojny), moyen-métrage de $33 \mathrm{mn}$ réalisé en 1972.

6. Coj mourra dans un accident de voiture en 1990.

7. Russia on Reels..., op. cit. 